\title{
PRÁCTICAS MATERNAS DE ALIMENTACIÓN E INCLUSIÓN SOCIAL DE NIÑOS MEXICANOS CON DISCAPACIDAD. APROXIMACIÓN CON GRUPOS FOCALES
}

\author{
Martha Elba Alarcón ${ }^{1, a, c}$, María del Refugio López,a, Magali Ramon,a, Irma Rosa Alvarado,a,d, Martha González ${ }^{1, b}$
}

\begin{abstract}
RESUMEN
Objetivos. El propósito del estudio fue identificar las prácticas maternas alimentarias con niños que presentan algún tipo de discapacidad y su influencia en el proceso de inclusión social. Materiales y métodos. Se realizaron tres grupos focales en los que participaron doce madres o cuidadoras de niños con discapacidad. Resultados. Las madres/ cuidadoras se ocupan de la mejoría de sus hijos respecto a su salud física y discapacidad. Sus prácticas de crianza alimentaria contribuyen a su estado de salud, al desarrollo de aprendizajes alimentarios, de comportamiento social en la mesa, de colaboración doméstica así como al desarrollo del lenguaje mediante las interacciones comunicativas y a estrechar los lazos afectivos madre-hijo. Conclusiones. Las prácticas de crianza alimentarias posibilitan la transmisión cultural de preferencias y aversiones alimentarias y adicionalmente, generan un contexto que promueve el desarrollo de aprendizajes de muy diversa índole, que para el caso de los niños con algún tipo de discapacidad, podrían coadyuvar a mejorar sus habilidades y repertorios prosociales mediados por las madres o cuidadoras.
\end{abstract}

Palabras clave: Alimentación; Relaciones madre-hijo; Grupos focales; Niño; Personas con discapacidad (fuente: DeCS BIREME).

\section{MATERNAL FEEDING AND SOCIAL INCLUSION PRACTICES IN MEXICAN CHILDREN WITH DISABILITIES. APPROACH WITH FOCAL GROUPS}

\begin{abstract}
Objectives. The aim of this study was to identify the maternal feeding practices with children who have some kind of disability and its influence in their social inclusion process. Materials and Methods. Three focus groups were carried out with the participation of twelve mothers/caregivers of children with disabilities. Results. The mothers/caregivers are in charge of their children's progress with respect to their physical health and disability. Their feeding practices contribute to their health condition, to the acquisition of nourishing learning, social behavior at the table, domestic collaboration, as well as to the development of language by means of the communicative interactions, and also the tightening of the affective bond between mother and child. Conclusions. Nourishing practices make possible the cultural transmission of food preferences and aversions and, additionally, generate a context that fosters the development of very diverse learning which, in the case of children with some type of disability, could contribute to the improvement of their pro-social skills and repertoires mediated by their mothers or caregivers.
\end{abstract}

Key words: Feeding; Mother-Child Relations; Focal groups; Child; Disabled Persons (source: MeSH NLM).

\section{INTRODUCCIÓN}

La alimentación es un proceso que abarca la selección de alimentos, su preparación, distribución de raciones e ingesta ${ }^{(1)}$. Este proceso que se repite cada día se transmite culturalmente a las nuevas generaciones y se modula o modifica en el trayecto de vida de los individuos por factores contextuales de la vida cotidiana (2). Además, posibilita diferentes aprendizajes a los nuevos miembros de la familia, no sólo aquellos relacionados con la selección de los productos que acostumbra consumir el núcleo social al que se pertenece, en función de su calidad y precio; se aprende cómo deben ser preparados, que utensilios emplear, los tiempos de cocción, sus combinaciones y

\footnotetext{
Facultad de Estudios Superiores Iztacala, Universidad Nacional Autónoma de México.

a Licenciada en psicología; ${ }^{\mathrm{b}}$ médica cirujana; ${ }^{\mathrm{c}}$ maestra en salud pública; ${ }^{\mathrm{d}}$ doctora en psicología.

Recibido: 24/03/2017 Aprobado: 07/02/2018 En línea: 05/04/2018
}

Citar como: Alarcón ME, López MdR, Ramon M, Alvarado IR, González M. Prácticas maternas de alimentación e inclusión social de niños mexicanos con discapacidad. Aproximación con grupos focales. Rev Peru Med Exp Salud Publica. 2018;35(1):32-8. doi: 10.17843/rpmesp.2018.351.3597. 
mezclas, así como también, la forma de presentarlos en la mesa y las cantidades pertinentes para cada miembro de la familia.

Por otro lado, a través de la alimentación se promueven comportamientos de carácter social, como modales en la mesa, patrones de higiene antes, durante y después de los alimentos, reglas de convivencia, de colaboración, normas y roles ${ }^{(3-5)}$. No obstante, los niños con discapacidad se encuentran con barreras que los excluyen de la vida social y que surgen en muchas ocasiones en el seno de las familias por falta de información de los padres, respecto a la discapacidad de sus hijos, actitudes negativas que los dejan fuera de actividades esenciales para su desarrollo e interacción con los demás y repercuten en la autoestima personal ${ }^{(6)}$

Los estudios que se han realizado respecto a la alimentación con individuos que presentan algún tipo de discapacidad son escasos y centrados en los trastornos de deglución y consumo en niños con diferentes tipos de discapacidad, principalmente motora ${ }^{(7-9,)}$; así como, en el examen de las alteraciones nutricionales en estas poblaciones ${ }^{(10-12)}$.

Regularmente las actividades e interacciones vinculadas a la alimentación, así como, su promoción y enseñanza, recaen en las madres, abuelas $u$ otros miembros femeninos integrantes de la familia o de empleadas que auxilian para estos casos a las familias. La mujer se constituye en una figura de protección y satisfacción de las necesidades de los menores desde el nacimiento y además es una fuente de socialización positiva ${ }^{(13)}$. Los niños aprenden regularmente de las mujeres todas las experiencias vinculadas alrededor de los alimentos y los productos para su preparación, sus aromas, texturas, sabores, consistencias, combinaciones y presentaciones en la mesa; asimismo, aprenden el uso de ciertos alimentos para las fiestas, las enfermedades o aquellos que son poco apreciados e incluso rechazados por la familia y en el contexto social del que se forma parte ${ }^{(5,14)}$.

Mantener silencio o conversar durante las comidas; utilizar una mesa o cualquier otro espacio para comer; colaborar o no antes, durante o después de las comidas dependerá de las normas y reglas familiares de la madre o cuidador en particular. De este modo, la alimentación se convierte en una actividad cotidiana socializante que integra a los niños al núcleo familiar primero y los va preparando para su inserción a contextos sociales más amplios donde se compartirán algunos o varios elementos ${ }^{(2,15)}$.

El proceso de alimentación juega entonces dos funciones principales en el desarrollo de los individuos, por un lado, se vincula a la salud al verse afectada por el estado nutricional del individuo generando susceptibilidad a ciertas enfermedades ${ }^{(16)}$, por el otro, es un factor muy relevante que lo prepara para la inclusión social ${ }^{(6,17,18)}$.

\section{MENSAJES CLAVE}

Motivación para realizar el estudio. Existe muy poca información acerca de las rutinas maternas relacionadas con la alimentación de los niños con discapacidad. Es necesario conocer las dificultades particulares para diseñar estrategias de intervención efectiva.

Principales hallazgos. Las madres se preocupan porque sus hijos consuman alimentos adecuados a su edad, sexo y condición de discapacidad y también procuran fomentar socialización de los infantes. Se observó que las madres responden a demandas adicionales de tiempo, organización de horarios y presentación de alimentos.

Implicancias. El conocimiento de las prácticas maternas de alimentación de niños con discapacidad puede ayudar a la formulación de políticas sanitarias y educativas para esta población.

Considerando lo anterior, el objetivo del estudio fue identificar las prácticas alimentarias maternas de menores con discapacidad que se vinculan a la inclusión social mediante una investigación cualitativa con grupos focales, que permite obtener información amplia respecto a las percepciones, formas de pensar y sentir de los individuos que los constituyen ${ }^{(19)}$

\section{MATERIALES Y MÉTODOS}

\section{DISEÑO Y ÁREA DE ESTUDIO}

El tipo de diseño fue etnográfico y descriptivo ${ }^{(20,21)}$ para explorar las prácticas de alimentación desarrolladas por las madres de niños con discapacidad asistentes al Servicio de Educación Especial de la Clínica Universitaria de Salud Integral Iztacala (CUSI), ubicada en el Estado de México y se efectuó en junio de 2015.

\section{PARTICIPANTES}

Se obtuvo la muestra por conveniencia ${ }^{(21)}$ y los criterios de inclusión de las madres fueron: a) aceptación para participar, b) tener al menos un hijo con discapacidad de entre tres y catorce años de edad, y c) que fueran las responsables de su alimentación. En caso de ausencia de la madre, se incluyó al cuidador más cercano. Participaron doce madres/cuidadoras de niños diagnosticados con algún tipo de discapacidad. Las características de madres y niños se muestran en la Tabla 1.

\section{ENTREVISTAS}

Se elaboró una guía temática con tres apartados para el desarrollo de los grupos focales. Los apartados de la guía fueron: a) costumbres para alimentar al niño, b) dificultades durante los periodos de alimentación del menor, y c) relaciones madre-hijo durante las comidas. 
Tabla 1. Características de madres e hijos por grupo focal

\begin{tabular}{ccccc}
\hline Participante & $\begin{array}{c}\text { Grupo } \\
\text { focal/participante }\end{array}$ & $\begin{array}{c}\text { Edad de la madre } \\
\text { (años cumplidos) }\end{array}$ & $\begin{array}{c}\text { Edad del niño } \\
\text { (años cumplidos) }\end{array}$ & Tipo de discapacidad \\
\hline 1 & GF1, P1 & 39 & 11 & Retraso psicomotor \\
\hline 2 & GF1, P2 & 40 & 12 & PEA \\
3 & GF1, P3 & 47 & 11 & PEA \\
4 & GF1, P4 & 36 & 8 & Retraso psicomotor \\
5 & GF1, P5 & 53 & 4 & Trastornos del lenguaje \\
6 & GF2, P1 & 51 & 8 & Síndrome de Down \\
7 & GF2, P2 & 47 & 6 & Retraso psicomotor \\
8 & GF2, P3 & 31 & 6 & Hipoacusia profunda \\
\hline 9 & GF3, P1 & 33 & 10 & PEA \\
10 & GF3, P2 & 47 & 8 & PEA \\
11 & GF3, P3 & & 12 & Síndrome de Lennox \\
\hline & & 30 & 10 & PEA \\
\hline
\end{tabular}

*Participantes con dos hijos en el servicio de educación especial. PEA: Problemas específicos de aprendizaje.

GF: Grupo focal

Los grupos focales fueron realizados en un aula de la CUSI en la Facultad de Estudios Superiores Iztacala bien iluminada y ventilada con una mesa al centro y sillas alrededor. Las participantes se sentaron formando una herradura. En cada sesión se encontraba presente el moderador y un auxiliar cuya función fue tomar notas y activar y desactivar la grabadora.

Los grupos focales se conformaron de acuerdo al horario de asistencia a la CUSI. Cada sesión inició después de que las madres/cuidadoras habían dejado a los niños en el salón o cubículo a cargo de su terapeuta. Cada grupo focal fue moderado ${ }^{(19)}$ por dos coordinadoras, una médica y una psicóloga, apoyadas por una observadora que tomaba notas y manejaba el equipo de grabación.

\section{ANÁLISIS DE DATOS Y ASPECTOS ÉTICOS}

Al inicio se solicitó autorización para grabar la sesión, se invitó a las participantes a firmar el consentimiento informado y a llenar un formato con los datos demográficos. Las sesiones tuvieron una duración aproximada de 45 minutos y se finalizó cuando se agotaron los puntos de la guía. Para el análisis de los resultados, se realizó la trascripción correspondiente, conservando en todos los casos la confidencialidad y anonimato de las participantes. Se recurrió a la triangulación de autores; se formaron tres equipos independientes que leyeron las transcripciones para hacer una primera aproximación, su posterior discusión y aprobación por el grupo de investigación.

\section{RESULTADOS}

Los resultados se organizaron en dos categorías de análisis a) prácticas maternas de alimentación, y b) vínculo con la inclusión social.

\section{PRÁCTICAS MATERNAS DE ALIMENTACIÓN}

Las madres se preocupan por la alimentación de sus hijos ya que por su discapacidad, de forma frecuente han estado en contacto con médicos y terapeutas. Para ellas es familiar lidiar con medicamentos y recomendaciones alimentarias aunque no necesariamente se encuentran bien informadas respecto a una correcta alimentación. Algunos de los hijos de las madres participantes consumen medicamentos como carbonato de magnesio, topiramato o gaspildor y refieren que para equilibrar estos medicamentos los niños deben comer "mucha verdura":

«... a él le tengo que dar mucha verdura verde porque él está tomando medicamentos fuertes, como es el carbonato de magnesio, como es el topiramato, gaspildor, entonces para equilibrar un poquito estos medicamentos tiene que comer mucha verdura verde» (GF1, P3).

"... pues pescado una vez al mes algo así por lo mismo ¿no? y este soy más de verdura, la carne por economía y por salud una vez a la semana... el pollo también una vez y ya lo demás es verdura» (GF2, P2).

«... yo diario hacía sopa de pasta... entonces se empezó a poner muy gordito, entonces diario es sopa pero ahora es sopa de verdura hoy es sopa de papa o sea si trato de quitar cosas que me dijo el nutriólogo que no lo nutren y que no más lo empanzonan, nos empanzonan y este yo siento que comen bien además gente me dicen es que come como un adulto no, y le dije come bien, nunca he tenido un problema así que se me caiga o se me desmaye por una anemia o algo así» (GF2, P1).

La preocupación por el sobrepeso sobre todo en los casos de síndrome de Down se manifiesta de forma repetida.

«A veces hasta le digo, ya no comas, porque a cada rato quiere estar comiendo, en el caso de mi hijo sí le pongo menos porque sé que tiende al sobrepeso por el síndrome de Down» (GF2, P1). 
«mi hijo tiene, no sé si el término es tiene, padece o sufre, no sé, pero tiene obesidad ... desde chiquito lo pusieron a dieta... siempre le he cuidado el peso» (GF3, P3).

En contraparte hay casos donde la madre observa pasivamente cuando el niño no ingiere o consume poco alimento a lo largo del día aunque lo atribuye a sus ocupaciones:

«No me quieren desayunar bien, no desayunan nada, comen tantito y se paran y ya no se lo acaban, me dejan la comida asi de caje, entera nada más sus tres cucharaditas y hasta ahí... y en la comida ellos comen igual, tres cucharaditas y se van a la escuela... pero en la cena arrasan con todo...por eso a veces no comen, [porque] yo ando ocupada, por eso nada más pellizcan la comida y comen solos» (GF1, P4).

La mayoría de las veces los menores son alimentados por sus madres o cuidadoras, regularmente en ausencia del padre por razones de trabajo o porque no vive con la familia. En los episodios de alimentación participan los hermanos o algún otro familiar como los abuelos o tíos aunque en algunos casos lo hacen solo con la madre porque conviven con ella la mayor parte del tiempo:

"Normalmente cuando la niña y yo ya llegamos comemos nosotras, pero cuando nos sentamos todos...a veces está abuelita, a veces tía» (GF3, P3).

El espacio que suelen utilizar para alimentar a los niños es principalmente la mesa, las madres mencionan que es el sitio para que sus hijos aprendan dónde comer.

«...porque ahí se debe comer» (GF1, P3).

También consideran que les permite crear hábitos de limpieza y orden:

"No puedes estar en la sala o en tu recámara y comer porque no, porque se ensucia, porque es un niño y tira moronas, entonces quiero que desde ahorita sepa que el lugar en donde se tiene que comer es en la mesa, en el comedor» (GF2, P2).

Asimismo, refieren a la mesa como un lugar cómodo, pues algunos niños requieren ayuda para alimentarse debido a su discapacidad. Algunas madres muestran disgusto si el niño ingiere alimentos en sitios distintos a la mesa:

"...porque me molesta que coma en la sala porque no es limpio para comer, no se acerca bien al plato entonces tira comida, o deja las servilletas tiradas o no se fija bien y tira el agua» (GF3, P1).

La preocupación en estas madres es la limpieza y probablemente el trabajo que implica para ellas:

«Principalmente porque puede tirar el vaso de agua o así no, y yo creo que más por ejemplo, porque lo dejo a la mejor pero después va a decir bueno pues puedo comer en la cama ¿no?, entonces no» (GF3, P1).

El comportamiento del niño durante los episodios alimentarios interesa a las madres por el posible impacto social en contextos diferentes al hogar.
«Si algún día van de visita a otra casa se deben de enseñar a comer en el lugar que debe»(GF1, P3).

Otras madres muestran mayor flexibilidad y permiten realizar la comida en la cama u otro lugar, para ellas, lo importante no es el lugar sino que se alimenten, inclusive, pueden comer en la cama viendo las caricaturas favoritas:

«Mi hijo a veces come viendo la televisión, le gusta ver las caricaturas, lo importante es que coma, ¿no?» (GF2, P3).

Los horarios de alimentación referidos por las madres son de rango amplio, el desayuno por ejemplo, fluctúa de seis a diez de la mañana, depende de las actividades del niño y la distancia que tienen que recorrer para asistir a la escuela o a la terapia:

«En mi caso el desayuno por cuestión de tiempo muchas veces se lo licúo...lo hago con el fin de que no venga con el estómago vacío»(GF2, P3).

«Se levantan a las siete y se toman un licuado» (GF1, P4).

El desayuno consiste principalmente de algo líquido y fácil de preparar:

«Toma leche», (GF1, P5).

"...por lo regular siempre trato de que desayunen cereal con leche $y$ yogurt con cereal $y$ fruta»(GF1, P1).

En el caso de la comida y cena el rango también es amplio: "Comemos entre tres y media y cuatro de la tarde» (GF3. P2).

"A la una le dan de comer porque se va a la escuela» (GF2, P2).

"La cena depende de la hora en que lleguemos a casa, puede ser a las diez o diez y media de la noche» (GF1, P3)

Algunas madres consideran insuficiente la ingesta de alimento por la mañana y preparan una colación para el niño. En otros casos se piensa que comer y cenar bien compensa la situación:

«Si, lleva lunch, fruta» (GF1, P5).

"Si, lleva de lunch una gelatina o sándwich, quesadillas y agua» (GF1, P1).

En otros casos, la colación no se contempla por razones que las madres consideran de peso como el comer bien previamente o porque el niño no la ingiere:

"Lunch no porque come bien en la casa» (GF1, P2).

«No les mando lunch porque me lo regresan o lo tiran» (GF1, P4).

Respecto a la última comida del día, las madres reportan una cena muy ligera e insuficiente.

«En la noche ya nada más se come su licuado» (GF3, P2).

«Cena cereal o lechita o galletitas» (GF2, P2).

Las porciones de alimento que ofrecen a los niños suelen ser pequeñas, se manifiesta la creencia de que los niños deben comer menos que los adultos: «Si, le damos porción de niño» (GF1, P5). 
"O sea, él quiere la misma porción que yo y yo le digo que no porque él no, aparte de que está muy niño, él no puede comer lo mismo que yo» (GF1, P3).

Unas madres preguntan a los niños que cantidad les sirven, pero dan raciones pequeñas:

"¿Cuánto vas a querer? no, poquito, ¿así? les enseño, y si quieren más ya les sivvo menor cantidad» (GF1, P4).

Pocas madres refieren usar la misma vajilla que el resto de la familia:

"Diario uso los mismos platos para todos... son mis platos de todos los días» (GF2, P3).

Las dificultades que mencionan las madres son diversas algunas por problemas masticatorios relacionados al tipo de discapacidad o problemas dentales:

"Él tiene síndrome de Down, él no mastica bien... a él le tengo que picar todo... desmenuzárselo, incluso yo la verdad, en donde esté, con la pena, yo me lavo las manos, o si no, nada más me las limpio y le desmenuzo todo» (GF2, P1).

Otra dificultad que enfrentan es cuando a los niños no les gusta la comida y lo expresan haciendo berrinche o dejando la comida:

"No se lo come y siempre la hace a un ladito y aunque esté chiquita la hace a un ladito y me dice guácala que no se la va a comer» (GF2, P1).

"A mi niña no le gustan mucho las verduras...las verduritas las va haciendo a un lado, me dice luego que eso no le gusta» (GF2, P2).

«Dice que no le gusta, no lo quiero y aunque le diga cómetelo pues dice que no» (GF2, $\mathrm{P} 2)$.

«Dice que no, "no mamá eso no me gusta» (GF3, P2).

"Con el chico si batallo porque le tengo que hacer el avión, cantar una canción, decirle que se lo hice con mucho amor de mamá y usar el chan...según yo el chantaje» (GF2, P3).

Los principales alimentos que no les gustan a los niños son las verduras en unos casos y la carne en otros:

«A mí niña, no le gusta mucho la verdura» (GF2, P2).

"La carne no es de su agrado» (GF2, P1).

Cuando a los niños no les gusta el alimento que les ofrecen sus madres estas despliegan alguna estrategia para intentar convencerlos de ingerirlo ya que les preocupa que no coman, por ejemplo, les dicen los beneficios o lo que puede pasarles:

«Si no te lo comes te puede pasar esto, esto y esto» (GF2, P3).

Otras sustituyen los alimentos:

«No quiere el huevito hoy, pues le doy una quesadilla" (GF2, P2).

"Trato de que mínimo se coma la mitad de lo que hay que comer, ya si la otra mitad no la come le pongo fruta» (GF2, P3).

«Lo mezclo con algo que sí le guste» (GF2, P1).

También las madres ponen de ejemplo al súper héroe favorito:
«Le tengo que inventar que se va a convertir en súper héroe o que va a cambiar de color como Hulk» (GF2, P3).

En otros casos, las madres retiran el alimento y los dejan sin comer hasta la siguiente comida:

"A ver, voy a levantar platos y el que comió, comió y el que no, ni modo» (GF3, P3).

También hay quienes los obligan a comer:

«Pues se la tiene que comer porque no hay otra» (GF2, P3).

Además de las aversiones o rechazo a determinados alimentos, se habla de las preferencias alimentarias de los hijos, en estos casos la dificultad es evitar que coman demasiado. Los alimentos que prefieren los niños son el arroz, frijoles, espagueti, huevo, lácteos y papa:

«Su comida preferida es el arroz con frijoles y crema, ahí se puede comer un kilo... y espagueti es lo que le encanta a mi hijo» (GF2, P1). «Le gustan más las quesadillas, este.... huevito» (GF2,P2). «...las papas a la francesa y los nuggets» (GF3, P3).

\section{VÍNCULO CON LA INCLUSIÓN SOCIAL.}

Las madres refieren el antes, durante y después de los episodios de alimentación. Se aprecia la preocupación por el aprendizaje, la formación de hábitos, la importancia de la cooperación familiar y el esfuerzo de logro:

"Yo tengo la idea de que él debe ganarse las cosas ¿no?, que los alimentos también deben ganarse, entonces pues lo involucro a que me ayude a poner la mesa, a que me ayude a lavar los trastes o a recoger o lo que sea» (GF1, P3).

"Ahorita le estoy enseñando a que se prepare sus alimentos, ya hace huevos revueltos, ya los prepara» (GF1, P3).

«Va a la cocina y empieza a poner las cosas» (GF1, P1).

Cuando hay rechazo de alguna actividad asignada, la madre sugiere algún cambio:

«bueno ok, tú no quieres trastes, bueno, tú prepara la cena y yo me pongo a lavar los trastes» (GF1, P3).

Otro aspecto relevante expresado en los grupos focales fueron las formas comunicativas de contenido más personal entre madres e hijos donde algunas veces las inicia el niño y otras la madre. Regularmente el contenido de estos intercambios comunicativos hace referencia al interés de saber respecto a otros miembros de la familia y a las actividades realizadas por el niño:

«Oye mamá y mi papá ¿por qué no está?» (GF3, P3).

«Oye mamá fijate que me pasó esto, esto y esto» (GF1, P1).

"¿Cómo les fue?, ¿Qué hicieron? ¿Con quién estuvieron?» (GF1, P2).

\section{DISCUSIÓN}

Los resultados muestran la preocupación de las madres porque sus hijos consuman alimentos en cantidad suficiente, acorde a la edad del niño; predisposición 
a la obesidad, como sucede en el caso de quienes son portadores de síndrome de Down; el efecto de los fármacos, principalmente anticonvulsivos, en la absorción de nutrimentos; o las dificultades particulares para masticar.

También conocen los beneficios para la salud que tiene el consumo de productos como frutas, verduras y carne y los ponen en práctica al cocinar o servir la comida, al respecto se observa que ellas despliegan una serie de prácticas para animar a sus hijos a comer y lograrlo, esto concuerda con lo encontrado en un estudio realizado en 2011 en Perú por el Instituto de Investigación Nutricional en una población de niños sin discapacidad ${ }^{(22)}$. Las estrategias utilizadas en algunos casos son de tipo autocrático y en otros el estilo es más democrático, pero en ambos casos se aprecia el mismo propósito. No obstante en ambos casos, en relación a la calidez/apoyo/afecto, se encuentran ambos estilos en un nivel bajo ${ }^{(5)}$.

Por otro lado, sólo algunas madres insisten en inducir la aceptación de los productos de menor agrado o franco rechazo, lo que limita convertir la rutina de alimentación en una actividad que incentive la adquisición o desarrollo de habilidades nuevas en el niño ${ }^{(23)}$, pero fortalecen preferencias y aversiones en los niños así como las percepciones relacionadas con el peso corporal ${ }^{(17)}$.

Aunque los episodios de alimentación constituyen también un espacio para desarrollar habilidades y comportamientos de cooperación, integración familiar y asignación de responsabilidades, estas se rigen por instrucciones específicas de la madre hacia el niño. En las interacciones comunicativas madre-hijo, ambos fungen como iniciadores a través de preguntas abiertas y aprovechan la permanencia en la mesa para mostrar su interés por las actividades realizadas en la escuela y terapia o por otros miembros de la familia.
Si bien, este estudio constituye un primer acercamiento a la relación entre prácticas maternas de alimentación y su vínculo con la inclusión social de infantes con discapacidad, es importante mencionar que una de sus principales limitaciones está dada por el hecho de que las madres provenían sólo de un servicio de educación especial, lo que reduce la generalidad de los hallazgos. En futuros estudios convendría abordar el problema con niños de edades y condiciones biológicas similares, lo que permitiría un análisis más profundo.

En suma las prácticas alimentarias de las madres se enfocan a la salud física de los niños y a impulsar la superación de su discapacidad promoviendo aprendizajes de comportamiento social, de colaboración doméstica así como al desarrollo del lenguaje mediante las interacciones comunicativas que estrecha los lazos afectivos madrehijo. Las madres a través de la alimentación promueven la inclusión familiar del niño mediante la asignación de actividades domésticas colaborativas, pero también, la inclusión a otros contextos cuando se promueve el comportamiento en la mesa. Los resultados obtenidos son un ejemplo más de que la alimentación, salud y educación recae principalmente en las mujeres, las madres son las responsables de cocinar, dar de comer, llevar a los hijos al médico o a terapia, desarrollar hábitos y comportamientos prosociales.

Contribuciones de los autores: MEA y MdRL concibieron el diseño del artículo y consiguieron el financiamiento. Todas participaron en la recolección de datos y contribuyeron a la redacción y revisión final.

Fuentes de financiamiento: Programa UNAM-DGAPA-PAPIIT.

Conflicto de intereses: los autores declaran no tener conflictos de interés.

\section{REFERENCIAS BIBLIOGRÁFICAS}

1. Bourges $\mathrm{H}$. La alimentación y la nutrición en México. Comercio Exterior [Internet]. 2001 [citado el 3 de febrero de 2017];51(10):897-904. Disponible en: http://revistas.bancomext.gob.mx/rce/ magazines/31/6/RCE.pdf

2. Busdiecker $S$, Castillo C, Salas I. Cambios en los hábitos de alimentación durante la infancia: una visión antropológica. Rev Chil Pediat. 2000;71(1):5-11. doi: 10.4067/S0370-41062000000100003.

3. Patrick H, Wiliams GC. Selfdetermination theory: its application to health behavior and complementary with motivational interviewing. Int J Behav Phys Act. 2012;9:18. doi: 10.1186/14795868-9-18

4. Díaz C, Gómez C. Sociología y alimentación. Rev Int Sociol. 2005;63(40):2146. doi: $10.3989 /$ ris.2005.i40.188
5. Black MM, Creed-Kanashiro HM. ¿Cómo alimentar a los niños? La práctica de conductas alimentarias saludables desde la infancia. Rev Peru Med Exp Salud Pública. 2012; 29(3):373-8. doi: 10.17843/rpmesp.2012.293.372

6. Organización Mundial de la Salud. El desarrollo del niño en la primera infancia y a discapacidad: un documento de debate. Ginebra: OMS; 2013.

7. Bacco JL, Araya F, Flores E, Peña N. Trastornos de la alimentación y deglución en niños y jóvenes portadores de parálisis cerebral: abordaje multidisciplinario. Rev Méd Clin Condes. 2014;25(2):330-42.

8. Leites ME. Abordaje de la alimentación y nutrición en niños con discapacidad. Rev Iberoam Estudios Psicomot y Tecs Corpor. 2007;25(1):53-62.
9. Johnson C, Turner K, Stewart P, Schmidt B, Shui A, Macklin E, et al. Relationships between feeding problems, behavioral characteristics and nutritional quality in children with ASD. J Autism Dev Disord. 2014;44(9):2175-84. doi: 10.1007/ s10803-014-2095-9

10. García L, Restrepo S. La alimentación del niño con parálisis cerebral un reto para el nutricionista dietista. Perspectivas desde una revisión. Perspect Nutr Humana. 2010:12(1):77-85.

11. Senna B, Campos L, Gottschall C. Nutritional assesment of children and teenagers with Down syndrome and congenital cardiopathy. Rev Chil Nut. 2012;39(2):151-8.

12. Águila A, Áibar P. Características nutricionales de niños con parálisis cerebral. 
ARIE-Villa El Salvador. An Fac Med Univ Repub Montev Urug. 2006;67(2):108-19. doi: 10.15381/anales.v67i2.1248

13. Betancourt L, Rodríguez M, Gempeler J. Interacción madre-hijo, patrones de apego y su papel en los trastornos del comportamiento alimentario. Universitas Médica. 2007;48(3):261-76.

14. Castrillón I, Giraldo O. Prácticas de alimentación de los padres y conductas alimentarias en niños: ¿Existe información suficiente para el abordaje de los problemas de alimentación? Revista de Psicología Universidad de Antioquia. 2014;6(1):57-74.

15. Bell R, Illán N, Benito J. Familia, escuela y comunidad: pilares para la inclusión. Revista Interuniversitaria de Formación del Profesorado. 2010;69(24,3):47-57.

16. Cordero, A. Principales enfermedades asociadas en el niño menor de un año. Medicent Electrón. [Internet]. 2014 [citado el 3 de febrero de 2017];18(3). Disponible en: http://scielo.sld.cu/pdf/ mdc/v18n3/mdc03314.pdf
17. Ogden, J. Psicología de la alimentación: comportamientos saludables y trastornos alimentarios. Madrid: Morata; 2005.

18. UNICEF, Ministerio de Educación, MINSAL, UNESCO, Ministerio del Trabajo, PNUD, FONADIS, OIT. Seminario Internacional: inclusión social, discapacidad y políticas públicas [Internet]. Santiago de Chile: Fondo de las Naciones Unidas para la Infancia; 2005 [citado el 3 de febrero de 2017]. Disponible en: http://unicef.cl/web/seminario-internacional-inclusion-social-discapacidad-y-politicas-publicas/

19. Hamui-Sutton A, Varela-Ruiz M. La técnica de grupos focales. Inv Ed Med. 2013;2(1):55-60.

20. Salgado Lévano AC. Investigación cualitativa: diseño, evaluación del rigor metodológico y retos. Liberabit. 2007;13(13):71-8.

21. Salamanca AB, Martín-Crespo C. El muestreo en la investigación cualitativa. Nure Investigación [Internet].2007 [citado el 3 de febrero de 2017];27. Disponible en: http://ceppia.com.co/Documentostematicos/INVESTIGACIONS O C IA L / MUESTRE O - IN V. CUALITATIVA.pdf

22. Creed-Kanashiro H, Penny ME, Carrasco M, Marin M. Estudio de línea de base situación de las prácticas de alimentación y nutrición materna infantil [Internet]. Lima: Care; 2011 [citado el 3 de febrero de 2017]. Disponible en: http://www. care.org.pe

23. García L, Restrepo S. Alimentar y nutrir un niño con parálisis cerebral. Una mirada desde las percepciones. Investigación Educativa de Enfermería. 2007;29(1):28-39.

Correspondencia: Martha Elba Alarcón Armendáriz

Dirección: Av. De los Barrios \#1, Colonia Los Reyes Iztacala, Tlalnepanlta, Estado de México, México.

Teléfono: 52(55) 56231333

Correoelectrónico:marthaaa@unam.mx

\section{Nuestros artículos se encuentran indizados en:}

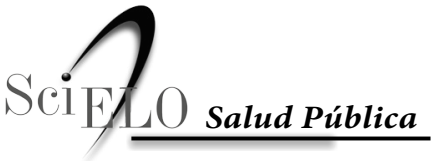

www.scielo.org 